As for the speed with which the limit in (b) is approached, we get, again using Taylor's expansion, the following:

II. In $\mathrm{I}(\mathrm{b})$, the error is $O\left(t^{3}\right)$. If $f$ has continuous fourth derivatives near $P$, the error can be made $O\left(t^{4}\right)$ by a further restriction on $S$. Whatever set $S$ is chosen, the error cannot be made $o\left(t^{4}\right)$ even if the class of functions is restricted to polynomials.

\title{
REFERENCES
}

1. L. Hopf, Differential equations of physics, Dover Publications, 1948, p. 62.

2. Garrett Birkhoff and David Young, Numerical quadrature of analytic and harmonic functions, J. of Math. and Physics 29, 217-221 (1950).

\section{A NOTE ON ASYMPTOTIC STABILITY*}

\section{By H. A. ANTOSIEWICZ (Montana State College)}

1. In this note we shall develop a stability criterion for a vector differential equation of the form

$$
\frac{d x}{d t}=A(t) x
$$

where the elements of the matrix $A(t)=\left(a_{i j}(t)\right), i, j=1,2, \cdots n$, are real continuous and uniformly bounded functions for all positive $t \geqq t_{0}$.

A. Wintner** recently established the following criterion: Let $\lambda_{1}(t)$ be the greatest, and $\lambda_{2}(t)$ the least characteristic value of the matrix $\frac{1}{2}\left[A(t)+A^{\prime}(t)\right]$, and let $\|x(t)\|$ denote the Euclidean length of the vector $x(t)$. If $\int^{\infty} \lambda_{1}(t) d t<\infty, \int^{\infty} \lambda_{2}(t) d t<\infty$, then $\|x(t)\| \rightarrow \kappa \neq 0$ as $t \rightarrow \infty$ for every non-trivial solution $x(t)$ of $(1)$.

It is to be noted that the condition of integrability of $\lambda_{1}(t), \lambda_{2}(t)$ over $\left(t_{0}, \infty\right)$ implies $\int^{\infty}$ [trace $\left.A(t)\right] d t<\infty$. Furthermore, this condition automatically excludes the important case $A(t)=$ const. unless $A(t)=$ const. is skew-symmetric.

In the following we shall establish a stability criterion which is free of the above objection, i.e. which will also apply to the general case $A(t)=$ const. We shall consider a condition to be satisfied by the matrix $A(t)$ which will suffice to insure that $\|x(t)\|$ of every non-trivial solution $x(t)$ of (1) tends to zero as $t \rightarrow \infty$. According to Liapounoff $\dagger$, the trivial solution $x(t) \equiv 0$ is then said to be asymptotically stable.

2. Consider a function $V(x, t)$ which is defined and continuous for all $x$ and $t$ in $R$ : $\left|x_{i}\right| \leqq c, t \geqq T(i=1,2, \cdots n)$. If for equation (1) there exists in $R$ a function $V(x, t)$ which is of fixed sign and admits of an infinitely small upper bound, and for which $d V / d t$ by virtue of (1) is opposite in sign to $V(x, t)$ in $R$, then the trivial solution $x(t) \equiv 0$ of (1) is asymptotically stable. Liapounoff proved that the existence of such a function

*Received April 16, 1951.

**A. Wintner, On free vibrations with amplitudinal limits, Quart. Applied Math. 8, 102-10: (1950).

$\dagger$ †. Liapounoff, Problème général de la stabilité du mouvement, Ann. Math. Studies, No. 17, 1949. 
$V(x, t)$ is sufficient for asymptotic stability; it is, however, not necessary as was shown by J. Malkin.*

We shall make use of Malkin's results to establish the following theorem:

Let $\lambda_{1}(t)$ be the greatest, and $\lambda_{2}(t)$ the least characteristic value of the matrix $\frac{1}{2}[A(t)+$ $\left.A^{\prime}(t)\right]$. If $\int^{t} \lambda_{1}(\tau) d \tau \rightarrow-\infty, \int^{t} \lambda_{2}(\tau) d \tau \rightarrow-\infty$ as $t \rightarrow \infty$, then $\|x(t)\| \rightarrow 0$ as $t \rightarrow \infty$ for every non-trivial solution $x(t)$ of $(1)$, i.e. the trivial solution $x(t) \equiv 0$ is asymptotically stable.

Note that now $\int^{\infty}[\operatorname{trace} A(t)] d t$ diverges.

3. First, we transform (1) into diagonal form. Let $x_{1}, x_{2}, \cdots x_{n}$ be a base of solutions of (1), and use this base to construct an orthogonal matrix $C(t)$. If $y=C^{-1}(t) x$, then (1) reduces to

$$
\frac{d y}{d t}=B(t) y, \quad B(t)=C^{-1} A C+\frac{d C^{-1}}{d t} C
$$

where the matrix $B(t)=\left(b_{i j}(t)\right), i, j=1,2, \cdots n$, is diagonal, i.e. $b_{i j}(t) \equiv 0$ for all $i>j$. If $y_{1}(t), y_{2}(t), \cdots y_{n}(t)$ is that base of solutions of (2) for which $y_{i}\left(t_{0}\right)=I^{i}$, the $i$-th column vector of the identity matrix $I$, then $\left\|x_{i}(t)\right\|=\left\|y_{i}(t)\right\|$ as is easily verified. Evidently, $C(t)$ and $C^{-1}(t)$ have bounded elements and $|C(t)|=\left|C^{-1}(t)\right|=1$; hence stability properties are preserved in both directions.

Observing that $C^{-1}(t)=C^{\prime}(t)$ by construction, we find by differentiating the identity $C(t) C^{-1}(t) \equiv I$ that $\left(d C^{-1} / d t\right) C$ is skew-symmetric. Therefore $B(t)+B^{\prime}(t)=C^{-1}[A(t)+$ $\left.A^{\prime}(t)\right] C$, and thus the characteristic values of $\frac{1}{2}\left[B(t)+B^{\prime}(t)\right]$ are identical with those of $\frac{1}{2}\left[A(t)+A^{\prime}(t)\right]$. Hence it is sufficient to prove our theorem for the reduced equation (2). We shall show that there exists a function $V(y, t)$ which satisfies Liapounoff's criterion for asymptotic stability.

Consider the diagonal elements $b_{i i}(t), i=1,2, \cdots n$, of the matrix $B(t)$. Since $\left(d C^{-1} / d t\right) C$ is skew-symmetric, trace $\left(d C^{-1} / d t\right) C \equiv 0$, and thus

$$
b_{i i}(t)=\left(C^{-1}\right)_{i} A C^{i}=\left(C^{i}\right)^{\prime} A C^{i}=\frac{1}{2}\left(C^{i}\right)^{\prime}\left[A(t)+A^{\prime}(t)\right] C^{i} .
$$

All diagonal elements of $B(t)$ are quadratic forms in the components of the column vectors $C^{i}$ of the matrix $C(t)$ for which we evidently have $\left\|C^{i}\right\|=1$. These quadratic forms attain their maximum and minimum on the unit sphere $\left\|C^{i}\right\|=1$ (compact set); if $\lambda_{1}(t)$ is the greatest, $\lambda_{2}(t)$ the least characteristic value of $\frac{1}{2}\left[B(t)+B^{\prime}(t)\right]$, then $\lambda_{1}(t)$ is the maximum, $\lambda_{2}(t)$ the minimum. From (3) we then obtain

whence for all $t \geqq t_{0}$

$$
\lambda_{1}(t) \geqq b_{i i}(t) \geqq \lambda_{2}(t)
$$

$$
\exp \left(\int_{t_{0}}^{t} \lambda_{1}(\tau) d \tau\right) \geqq \exp \left(\int_{t_{0}}^{t} b_{i i}(\tau) d \tau\right) \geqq \exp \left(\int_{t_{0}}^{t} \lambda_{2}(\tau) d \tau\right) .
$$

By hypothesis $\int^{t} \lambda_{k}(\tau) d \tau \rightarrow-\infty$ as $t \rightarrow \infty, k=1,2$, and thus

$$
\varphi_{i}(t)=\exp \left(\int_{i_{0}}^{t} b_{i i}(\tau) d \tau\right) \rightarrow 0 \quad \text { as } \quad t \rightarrow \infty .
$$

As Malkin has shown, (6) involves for all $t \geqq t_{0}$

$$
\varphi_{i}(t) \int_{t_{0}}^{t} \frac{d \tau}{\varphi_{i}(\tau)} \leqq c
$$

*J. Malkin, Certain questions on the theory of the stability of motion in the sense of Liapounoff, American Math. Soc., Translation No. 20, 1950. 
and (6) and (7) together, in turn, imply $\int^{\infty}\left[\varphi_{i}(t)\right]^{2} d t<\infty$. Hence the functions

$$
\psi_{i}(t)=\left[\varphi_{i}(t)\right]^{-2} \int_{t}^{\infty}\left[\varphi_{i}(\tau)\right]^{2} d \tau
$$

exist for all $t \geqq t_{0}$ and are uniformly bounded; in fact, $a^{2} \leqq \psi_{i}(t) \leqq b^{2}$ where $a$ and $b$ are certain constants.

Now consider the function

$$
V(y, t)=\psi_{1}(t) y_{1}^{2}+\psi_{2}(t) y_{2}^{2}+\cdots+\psi_{n}(t) y_{n}^{2} .
$$

It evidently satisfies Liapounoff's criterion for asymptotic stability; it is a positive definite quadratic form, admitting of an infinitely small upper bound, and its derivative, by virtue of (2), becomes

$$
\frac{d V}{d t}=-\left(y_{1}^{2}+y_{2}^{2}+\cdots+y_{n}^{2}\right)+W(y, t)
$$

where $W(y, t)$ is a quadratic form whose coefficients depend upon those elements $b_{i j}(t)$ of $B(t)$ for which $i<j, i, j=1,2, \cdots n$. Since these elements can always be made sufficiently small by a transformation with constant coefficients (which will not affect stability properties) the derivative $d V / d t$ will be a negative definite quadratic form. Hence the trivial solution $y(t) \equiv 0$ of $(2)$ is asymptotically stable, and therefore the trivial solution $x(t) \equiv 0$ of $(1)$ is asymptotically stable. This establishes our theorem.

O. Perron* was the first to prove directly that the conditions

$$
\varphi_{i}(t) \leqq C_{1}, \quad \varphi_{i}(t) \int_{t_{0}}^{t} \frac{d \tau}{\varphi_{i}(\tau)} \leqq C_{2}
$$

are necessary and sufficient for the trivial solution $x(t) \equiv 0$ of (1) to be asymptotically stable.

*O. Perron, Die Stabilitaetsfrage bei Differentialgleichungen, Math. Zeitschrift 32, 703-728 (1930).

\section{CONDITIONS SATISFIED BY THE EXPANSION AND VORTICITY OF A VISCOUS FLUID IN A FIXED CONTAINER*}

By J. L. SYNGE (Dublin Institute for Advanced Studies)

1. Introduction. In plane motion of a viscous fluid inside a fixed container, the expansion $\theta$ and the vorticity $\omega$ cannot be arbitrarily assigned. A necessary and sufficient condition ${ }^{1}$ for the consistency of given $\theta$ and $\omega$ with vanishing velocity on the walls is

$$
\int(\theta U+2 \omega V) d S=0
$$

*Received April 17, 1951. This paper was written while the author was on leave of absence at the Institute for Fluid Dynamics and Applied Mathematics, University of Maryland.

1J. L. Synge, Quarterly of Applied Mathematics, 8, 107-108 (1950). The condition with $\theta=0$ was originally due to G. Hamel, Göttinger Nachr. Math.-Phys. Kl. 1911, 261-270. 